\title{
Las actividades enfermeras en la consulta de enfermedad renal crónica avanzada contribuyen a mantener la función renal
}

\author{
Francisco Cirera Segura, Macarena Reina Neyra, Álvaro Pérez Baena, Jesús Lucas Martín Espejo
}

\section{Unidad de Gestión Clínica Uro-Nefrológica. Hospital Universitario Virgen del Rocío. Sevilla}

\section{Introducción:}

En España la población mayor de 65 años se ha duplicado en los últimos 30 años, aumentado la incidencia de enfermedad renal crónica avanzada (ERCA). Aunque esta variable no se puede modificar, existen otras relacionadas con el estilo de vida que mediante intervenciones enfermeras en la consulta ERCA, podemos realizar para retrasar el deterioro de la función renal (FR). Mantener la FR ha demostrado mejorar la supervivencia de estos pacientes, y aunque la tasa de filtración glomerular es el mejor índice para valorarla, no es fácil medirlo en la práctica clínica; por esto se utilizan diversas ecuaciones. Nuestro objetivo fue valorar el deterioro de la FR en la consulta ERCA, para determinar si las actividades enfermeras que desarrollamos contribuyen a su mantenimiento. El objetivo secundario fue comparar el deterioro de la FR mediante dos fórmulas distintas.

\section{Material y método:}

Se realizó un estudio descriptivo retrospectivo, de 6 meses de duración, cuya muestra fueron 148 pacientes. Se recogieron datos demográficos, analíticos, epidemiológicos. La FR se evaluó mediante el aclaramiento de creatinina, la ecuación Modification of Diet in Renal Disease (MDRD) y la ecuación del grupo Chronic Kidney Disease Epidemiology Collaboration (CKD-EPI). EI análisis de datos se realizó con el SPSS 19.0.

\section{Resultados:}

La muestra la formaron 148 pacientes, $60,8 \%$ hombres. La edad media fue $68,76+14,85$ años. La etiología de la enfermedad renal más común fue la nefropatía diabética $(18,2 \%)$ y la vascular (16,9\%). El $45,3 \%$ eran diabéticos. Estudiamos la FR según la etiología, siendo mayor el deterioro en la glomerulonefritis $(-3,93)$ y la nefritis intersticial $(-3,22)$, aunque sin existir diferencias significativas $(p=0,286)$. Las vasculares fueron en las que menos se deterioró $(+0,19)$. Conseguimos disminuir el peso de los pacientes significativamente $(p<0,001)$ y el IMC $(p<0,001)$, a pesar de lo cual al final fue de $29,74+5,76$. Sólo nueve pacientes normalizaron su IMC, deteriorándose menos la FR en este subgrupo $(0,12$ vs $-2,16)$, aunque no se encontraron diferencias significativas $(p=0,474)$. Tanto los edemas $(26,1 \%$ vs $18,8 \%$ ) como el uso de diuréticos disminuyeron durante el estudio, aun manteniendo la diuresis $(p=0,751)$. Analizamos la evolución del daño renal en el tiempo, no observamos cambios en la proteinuria $(p=0,309)$ aunque sí las encontramos en el cociente albuminuria/creatinina ( $p=0,023$ a los 3 meses; $p=0,037$ a los 6 meses) y en la creatinina sérica $(p=0,003 ; p<0,001)$ Evaluamos el deterioro de la FR medido a través del aclaramiento de creatinina y dos fórmulas distintas. Obtuvimos diferencias significativas para aclaramiento de creatinina $(p=0,006)$, MDRD $(p=0,003)$ no siendo así para CKP$\operatorname{EPI}(p=0,487)$.

\section{Discusión:}

Mediante las intervenciones de Enfermería realizadas, conseguimos mantener la diuresis disminuyendo los edemas y los diuréticos y el peso y el IMC descendieron. Aunque se produjo un deterioro significativo en algunos de los factores de daño renal, no tuvieron relevancia clínica, pudiendo afirmar que las actividades desarrolladas en la consulta ERCA pueden contribuir al manteni- 
miento de la FR. Existieron diferencias significativas al valorar el deterioro de la FR mediante las dos fórmulas, ya que la ecuación MDRD puede subestimar la FR.

\section{Referencias Bibliográficas}

1. Instituto Nacional de Estadística, INE [acceso 23 febrero 2013]. Disponible en: htt://www.ine.es.

2. Gorostidi $M$, Alonso JL, González de Cangas $B$, Jiménez $F$, Moína MJ, Vega $C$. Prevalencia de insuficiencia renal en población de edad avanzada y factores asociados. Resultados preliminares. XXXVI Congreso Nacional de la SEN. Resumen en Nerfología 2004; 24 (Supl. 6).

3. Bardón E, Marti i Monros A, Vila M ${ }^{a}$ L. Enfermería en la consulta de enfermedad renal crónica avanzada (ERCA). Nefrología. 2008; (Supl. 3): S53-S56.

4. Caravaca F, Cid MC, Galán J, García MC Cubero $\mathrm{JJ}$, Arrobas $\mathrm{M}$ et al. Relación entre el aclaramiento de urea y la tasa de catabolismo proteico en pacientes en DPCA: importancia de la función renal residual. Nefrología. 1996;(16):228-35.

5. Rodríguez P, Gómez FJ. Importancia de la función renal residual en pacientes en hemodiálisis. Nefrología 2002; (22)2: 98-103.

6. Gómez MA, Rodríguez E, Recio JI, Martín C, Ramos R, García L. Diferencias de la ecuación CKDEPI con la de MDRD para la estimación del filtrado glomerular en pacientes hipertensos. Nefrología. 2010; 30(4):458-62.

7. Coronel F, Cigarrán S, Herrero JA, Martín P. Función renal medida por GFR-MDRD-7 en diabéticos al iniciar diálisis peritoneal y su influencia en la supervivencia. Nefrología 2004; 24 (V): 53.

8. Molina MA, Ruiz MC, Alaguero B, Gómez B, Rodrigo $A$, De Paula $A$ et al. Consulta de enfermedad renal crónica ¿Cómo lo estamos haciendo...? Nefrología. 2004; 24(V):30.

9. National Kidney Foundation. K/DOQI clinical practice guidelines for chronic kidney disease: evaluation, classification, and stratification. Am J Kidney Dis 2002; 39(Supl. 1):S46-S75.

10. Montañés Bermúdez R, Bover Sanjuán J, Oliver Samper A, Ballarín Castán JA, Gràcia García S. Valoración de la nueva ecuación CKD-EPI para la estimación del filtrado glomerular. Nefrología. 2010;30(2):185-194.

11. Gracia S, Montañés R, Bover J, Cases A, Deulofeu R, Martín de Francisco AL, et al. Documento de consenso: Recomendaciones sobre la utilización de ecuaciones para la estimación del filtrado glomerular en adultos. Nefrología 2006; 26(6):658-66.

12. Caravaca F, Arrobas M, Luna E, Pizarro JL, García C, Espárrago JF et al. Progresión de la insuficiencia renal en pacientes con enfermedad renal crónica avanzada. Nefrología. 2003; 23(6):510-19.

13. Puerta M, Kanter J, Goicoechea M, García de Vinuesa S, Gómez-Campderá F, Luño J. ¿Qué características tienen los pacientes que acuden por primera vez a consultas externas de nefrología? Nefrología 2005; 25 (Extra 4):4-6.

14. Locatelli F, Del Vecchio L, Andrulli S, Marai $P$, Tentori $F$. The role of underlying nephropathy in the progression of renal diseases. Kidney Int 2000; 75:S49-S55. 